\title{
Improvement of Mechanisms for Network Time Synchronization Algorithm Based on Wireless Sensor Network
}

\author{
Lin Zhou ${ }^{1, \mathrm{a}}$, Jialun $\mathrm{Li}^{2, \mathrm{~b}}$, Longpin Yang ${ }^{3, \mathrm{c}}$ \\ ${ }^{1,2}$ College of Communication and Information Engineering, Chongqing University of \\ Posts and Telecommunications, Chongqing 400065, China \\ ${ }^{3}$ Beijing Hostipal, Beijing 100005, China \\ aemail:1058443685@qq.com, bemail:1241333521@qq.com,'cemail:825908296@qq.com
}

Keywords: WSN; TimeSynchronization; RBS; Clustering

\begin{abstract}
TimeSynchronization is one of the most important support Technology in WSN,plays an irreplaceable role in the developmet of WSN.For this reason,an improved algorithm based on theRBS algorithm is proposed combination of some classic time synchronization algorithm.Referring to the idea of cluster-based, the introduction of Synchronization mechanism for both cluster-head and cluster-within, ensure the synchronous accuracy and save the energy consumption.By comparing the experiment results, the improved mechanism reduce communication overhead.
\end{abstract}

\section{Introduction}

Time synchronization in wireless sensor networks means all or part of the network nodes have the same time reference, which means Different nodes maintains the same clock[1].Time synchronization plays a crucial role in the applications of wireless sensor network. However different applications in WSN have different requirement for time Synchronization, such as:accuracy, longevity, scope, cost, energy and other. after years of hard efforts, a variety of time synchronization algorithm have been propoesd,they solve the time synchronization problem with Different methods,and the improved RBS protocol in this paper is the synchronization algorithm based on the receiver-receiver[2], and the main goal is to ensure the synchronization accuracy,reduce communication overhead and information exchange capacity.

\section{RBS Protocol}

Reference Broadcast Synchronization (RBS)takes advantage of the characteristics of broadcast channel in the wireless data link layer,reference node periodically send reference broadcast to other neighbors node in the network,the neighbor nodes record the local time when they received broadcast,use it as the reference point compared with the clock readings[3].In order to calculate the clock skew,the timestamp between the equivalent neighbor need to be exchanged,so will determining the offset between them. Then one of those node change it's local time according to the timestamps[4],in order to achieve time synchronization.

Calculated as follows:

$$
\forall i \in n, j \in n: \text { offset }[i, j]=\frac{1}{m} \sum_{k=1}^{m}\left(T_{j, k}-T_{i, k}\right)
$$

Where,n represents the number of recipients,m represents the number of reference packets, $\mathrm{T}_{\mathrm{rb}}$ represents the clock when the receiving node $\mathrm{r}$ receiving the reference packet $\mathrm{b}[5]$. 


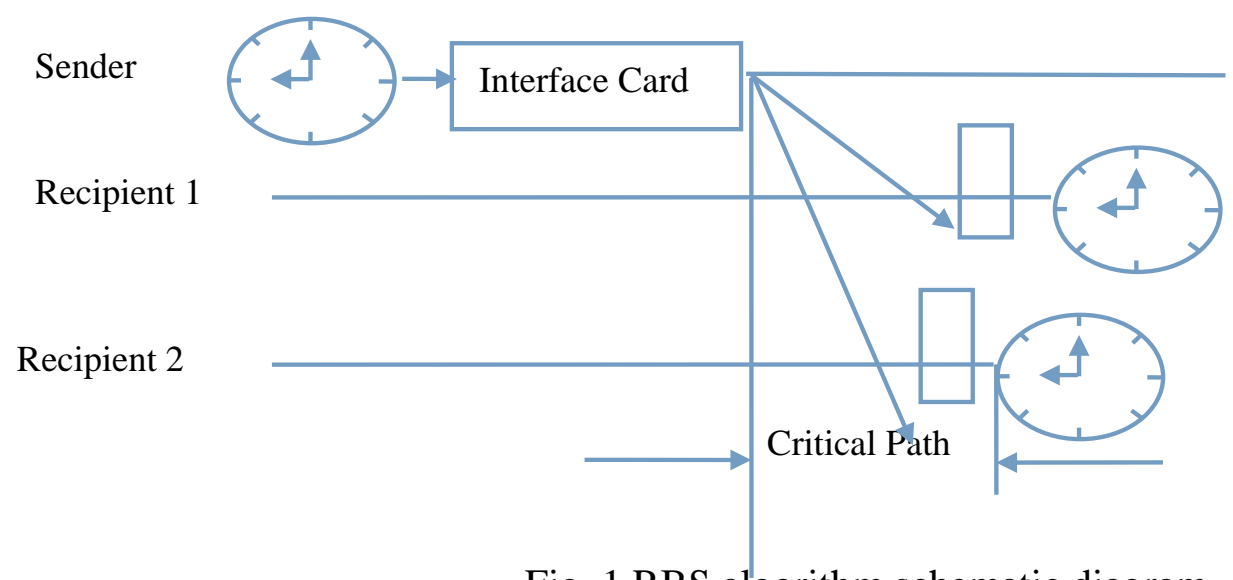

Fig. 1 RBS algorithm schematic diagram

Analysis of algorithm.This an algorithm that allows receivers synchronize with each other,which effectively avoid the influence of access time on synchronization,remove the uncertainty of the sender's delay from the critical path.The main reason of error come from the uncertain of transmission time and receive time,so compared to the Bidirectional information exchange between nodes[6],this algorithm obtain higher accuracy.As to it's biggest drawback is too many information exchange times.High computational complexity,network traffic overhead and energy consumption is too large,all these make it not suitable for the occasion of limited energy supply[7].

\section{Improvement of RBS Mechanism}

Clustering is the core idea of this algorithm,there ara two steps:firstly,the base station send broadcast beacon to all cluster heads, base station and cluster heads will achieve synchronization,secondly cluster heads broadcast to Intra-node clusters,so all nodes within the cluster will be synchronized.The energy of the cluster-head node ,the distance between cluster-head node and Intra-node cluster are all the factors should to be considered in the process of cluster head election. There are two forms to achieve synchronization in synchronous phase:firstly,Bidirectional synchronous principle will be taken in the process of time synchronization between base station and cluster heads,so the transmission time between the base station and the clock skew can be calculated,the cluster head will adjust it's own clock according to the two parameters,so the synchronization between base station and cluster head is complete.secondly,the cluster head send broadcast to all the Intra-node cluster in the same cluster,then the Intra-node clusters exchange information with each other use RBS mechanism for reference,after comparison with each other,in order to achieve synchronization within the cluster.

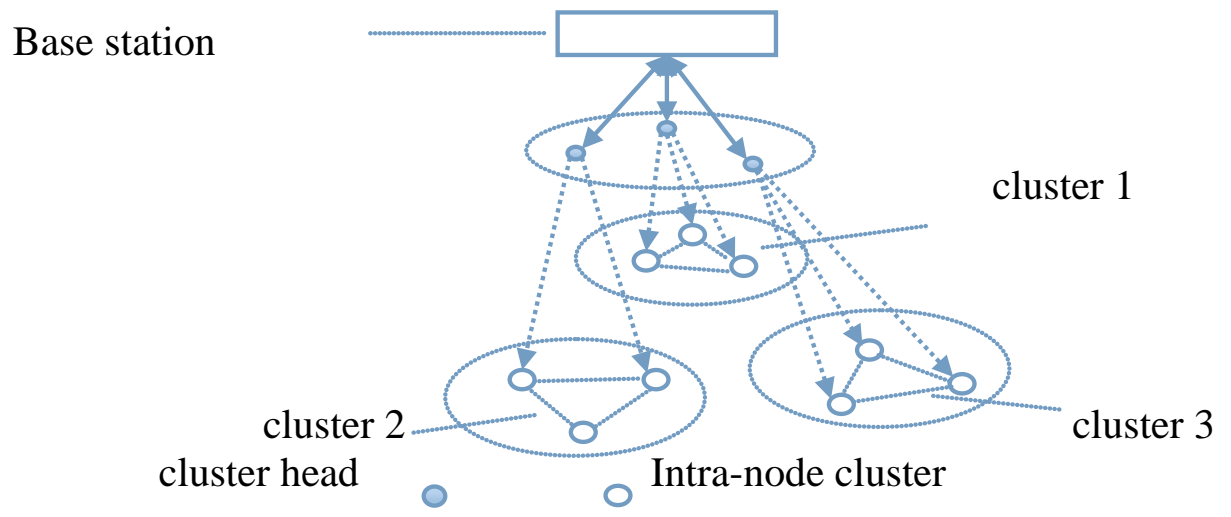

Fig.2 Improved algorithm topology 
Principles of the synchronous process

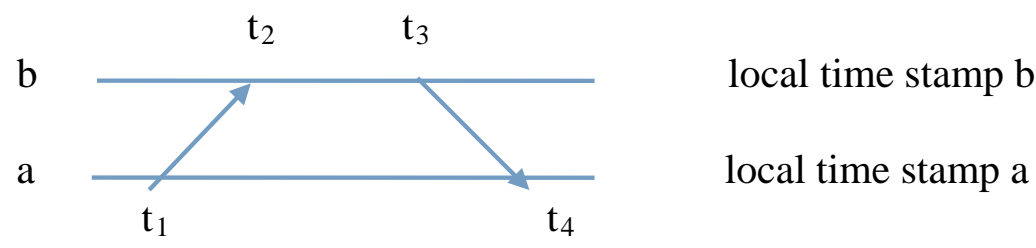

Fig.3 Bidrectional synchronous principle

Bidirectional synchronous principle as shown in Fig.3,a and b represent the two nodes, $\mathrm{t}_{1}$ and $\mathrm{t}_{4}$ represent the different time measurements of local clock of node $a, t_{2}$ and $t_{3}$ represent the different time measurements of local clock of node b.D represents the time offset between two nodes, $d$ represents the information transmission delay.By default,one message has the same transfer delay in the process of transmission.By using the above parameters,node a can calculate time offset $\mathrm{D}$ and information transmission delay d,so node a will adjust it's own clock to achieve synchronization.

$\mathrm{D}=\left(\left(\mathrm{t}_{2}-\mathrm{t}_{1}\right)-\left(\mathrm{t}_{4}-\mathrm{t}_{3}\right)\right) / 2$
$\mathrm{~d}=\left(\left(\mathrm{t}_{2}-\mathrm{t}_{1}\right)+\left(\mathrm{t}_{4}-\mathrm{t}_{3}\right)\right) / 2$

Then start the synchronization within a cluster,synchronization mechanism has similar principle with RBS algorithm.All cluster head has been synchronization because of the first stage.Each cluster head send broadcast beacon to the nodes within their own clusters.Intra-node cluster record the local time when they received broadcast,use it as the reference point compared with the clock readings,the timestamp between the equivalent nodes need to be exchanged,so will determining the offset between them,will synchronization be completed.Calculated as formula 1.

\section{Simulation Results and Analysis}

Communication cost is a very important indicator of time synchronization in the WSN.The RBS algorithm and the improved algorithm are compared by simulation in this paper.The simulation environment is a square area of $200 \mathrm{~m} \times 200 \mathrm{~m} \cdot 100,150,250,300$ static nodes are deployed randomly,nodes communication distance is set to $25 \mathrm{~m}$.

Through simulation,we found that the communication cost of the improved algorithm is much less than the RBS algorithm. This is because each node has only to receive and send a message within a cluster,and the cluster heads use Bidirectional synchronous mechanism are much less than the total. (It can be seen from Fig.4 )

Another important indicator is the information exchange capacity,as we can see from the Fig.5.With the increase in the number of nodes, the information exchange capacity of improved algorithm significantly lower than the RBS algorithm under the same number.This because each node under the base station will exchange information with the rest nodes in the RBS algorithm,but for the improved algorithm,only cluster heads need change information with base station in the first stage,and for the second stage,information exchange capacity within each cluster is much less.So the total amount of capacity will be reduced. 


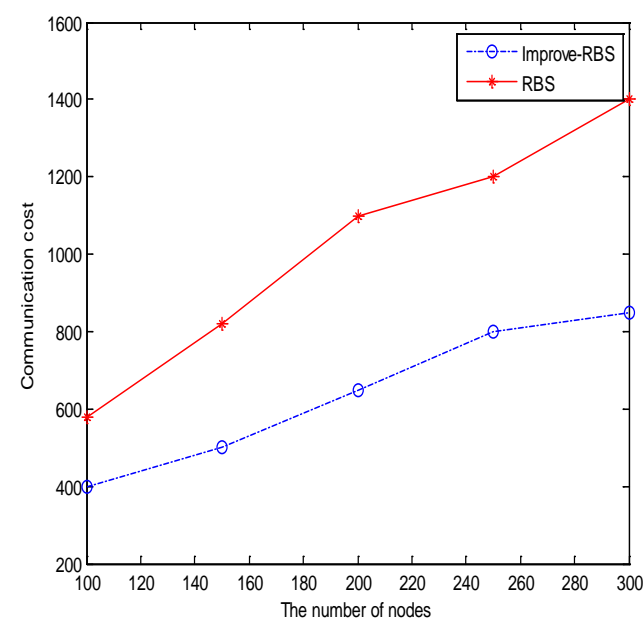

Fig.4Communication cost simulation map

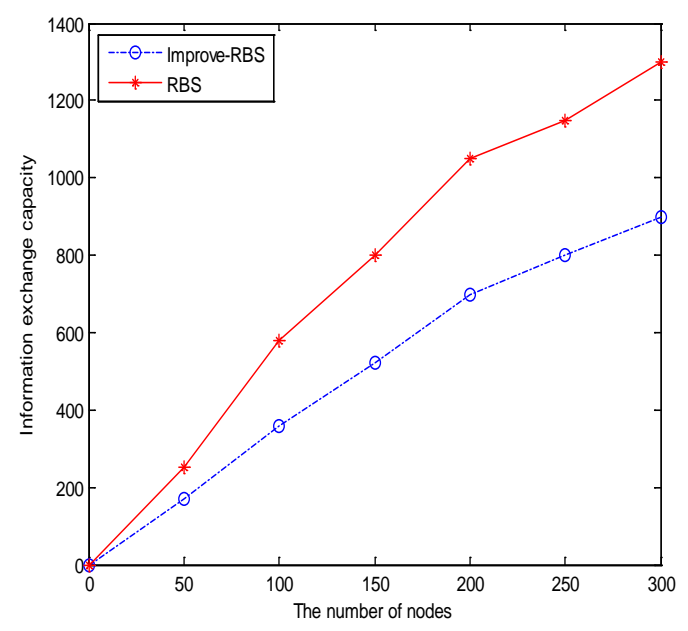

Fig.5Information exchange capacity simulation map

\section{Conclusion}

For the time synchronization in wireless sensor network,this paper presents an improved algorithm based on the RBS algorithm,introducing clustering mechanism.Considering the cost of energy and exchange capacity,by comparison the improved algorithm can improve the energy consumption and the exchange capacity.

\section{Ackowledgement}

This research was financially supported by National Natural Science Foundation of China(Grant No.61171190)

\section{References}

[1] Xu Chaonong, Zhao Lei, Xu Yongjun,et al1A time synchro-nization improvement strategy for wireless sensor networks[J]1Chinese Journal of Computers, 2007, 30(4): 514-523 (in Ch-inese).

[2]I.F.Akyildiz,W.Su,Y.Sankarasubramaniam,E.Cayirinc”A Survey on Sensor Networks, IEEE Communications Magazine,vol.40,no.8,pp.102-114,2002.

[3] Rhee I K, Lee J, Kim J, et al. Clock Synchronization in WirelessSensor Networks: An Overview [J]. Sensors, 2009, 9(1): 56-85.

[4] K.-L. Noh, E. Serpedin, and K. Qaraqe, A new approach for time synchronization in wireless sensor networks:pair wise broadcast synchronization, IEEE Trans. Wireless Communication., vol. 7, no. 9, Sep. 2008.

[5] Elson J, Romer K. Wireless sensor networks: a new regime for time syn chronization[C]. Proceedings of the 1st Workshop on HotTopics In Networks ( HotNet s- I), Princeton, New J ers ey, 2002:28- 29

[6] Liu Linf eng, Liu Ye. A heuristic cluster controlal gorithm of wireless sensor networks topology [ J] . Journ al of ComputerResearch and Development , 2008 ( 7 ) : 1099-1105 ( in Chinese).

[7] R6merK,BlumP,MeierL.Time Synehronization and CalibrationinWireless Sensor Networks. Wireless Sensor Neorks,IvanStojmenovie,ed.2005. 